\title{
A Novel Approach to Provide Max Flow in WSN: A Review
}

\author{
Vimandeep $^{1}$, Ms. Sonam Khattar ${ }^{2}$ \\ Computer Science \& Engineering Dept, Galaxy Global Group of Institutes, Kurukshetra ${ }^{1,2}$
}

\begin{abstract}
The main challenge in wireless sensor network deployment pertains to optimizing energy consumption when collecting data from sensor nodes. The routing protocols are the hot areas to address quality-of-service (QoS) related issues viz. Energy consumption, network lifetime, network scalability and packet overhead. The key issue in WSN is that these networks suffer from the packet overhead, which is the root cause of more energy consumption and degrade the QoS in sensor networks. We further present a meta-heuristic Wireless Sensor Networks Tabu Search (WSN-TS) algorithm to address the NP-completeness of the channel assignment problem. The main aim is to provide extra energy to nodes that helps to provide maximize flow rate at the sink. The energy constraint ensures the amount of recharged energy for each node is larger than its consumed energy.
\end{abstract}

Keywords: WSN System, Routings in WSN, Tabu Search, QoS in WSN etc.

\section{INTRODUCTION}

WSN term can be generally sensed as devices range from laptops, PDAs or mobile phones to very tiny and simple sensing devices. At present, most available wireless sensor devices are considerably controlled in terms of computational power, memory, efficiency and communication capabilities due to economic and technology reasons. That's why most of research on WSNs has focused on the design of energy and computationally efficient algorithms and protocols, and the application domain has been confined to simple data oriented monitoring and reporting applications. WSNs nodes are battery powered which are organized to perform a specific task for a long period of time, even years. If WSNs nodes are more powerful or mains-powered devices in vicinity, it is beneficial to exploit their computation and communication resources for complex algorithms and as gateways to other networks. New network designs with heterogeneous devices and expected developments in technology are eliminating current limitations and expanding the spectrum of possible applications for WSNs considerably.

The operation of a WSN is tightly linked to its unpredictable deployment environment. Obviously, the environment determines sensor readings on the nodes, but it can also affect other, interlinked properties like communication delay, energy consumption and network topology. The context for WSNs can vary considerably and some situations can be completely unknown to the developer. Sensor nodes in WSNs are small sized and are capable of sensing, collecting and processing data while communicating with other connected nodes in the network, via radio frequency (RF) channel [1]. The topology of the WSNs can vary from a simple star network to an advanced wireless mesh network. The propagation technique among the nodes of the network could be routing or flooding. The power of the wireless sensor networks lies in the capability to deploy large numbers of small nodes that assemble and configure themselves. In addition to drastically decreasing the installation costs, wireless sensor networks have the capability to dynamically adapt to changing environments. Adaptation mechanisms can lead to changes in network topologies or can cause the network to shift between different modes of operation.

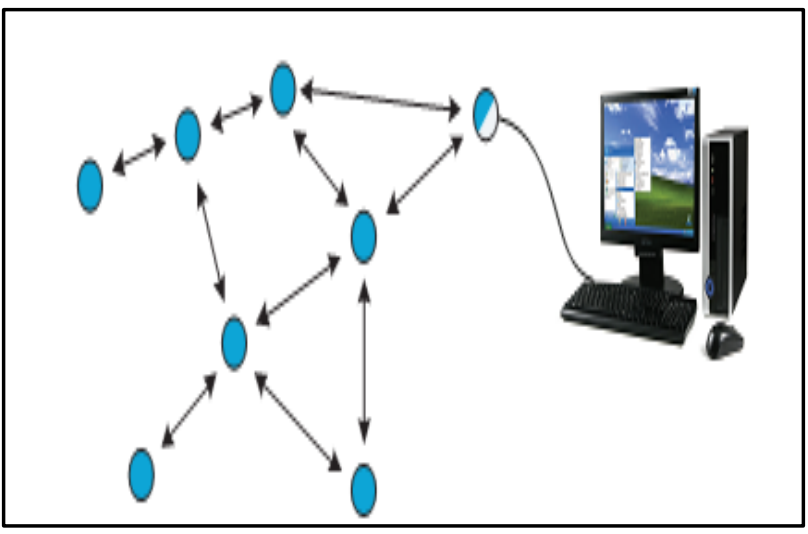

Figure 1.1: Wireless Sensor Network [1] 
The information coming from the WSN is generated by sensors on board the nodes. Using micro electro mechanical systems (MEMS) and analog-to-digital converters, values of various physical phenomena can be measured and converted to a binary representation. The sensitivity, accuracy and sampling rate can all be configured by the user. To avoid excessive communication, sensor data is often processed locally before being made available to the rest of the network. For exchanging information, nodes are equipped with a wireless radio. The transmission range is limited and varies unpredictably over time and space, because of physical phenomena like signal reflection, diffraction, shadowing and background noise.

The paper is ordered as follows. In section II, it represents related work of proposed system in WSN network. In Section III, It defines the various routing protocols. Section IV defines the concept of tabu search. The proposed system is defined in section V. Finally, conclusion is explained in Section VI.

\section{RELATED WORK}

Jaspreet kaur et . al.[1] developed a Tabu Search, Energy Efficiency, Clustering, Data Aggregation, ERA, WSNs. Data aggregation at the bottom station with entity nodes act as the root for overflow of the information which consequences in peak power utilization. To diminish this dilemmaa new data aggregation technique has been proposed called ER which has improved the performance of the WSNs by using the group based data aggregation but even then it has some limitations. The compressive sensing uses data fusion to remove redundant data from sensor nodes, so improves the results further. Era has shown quite significant consequences over the different available protocol of wireless sensor network.

Meng husi jeo et.al [2] proposed Wormhole attacks, Wireless Sensor Network, Quantum-inspired Tabu Search Algorithm, Moving Average Wireless sensor networks (WSN) can be widely used in many areas, such as environment monitoring, weather forecasting, traffic control, etc. The wormhole attack problem is an important issue in WSN since it causes many problems, such as routing error, a reduction in sensor lifetime and broken network topology. Several wormhole detection approaches have been proposed, but most of them need special hardware devices and consume a lot of system resources.

Rava chammas et.al.[3] developed a Wireless sensors network; sensor placement; grid network; detection probability; coverage; tabu search. Sensor placement is a crucial task for any wireless sensor network (WSN) application. Sensor placement has a direct impact on the efficiency of any WSN application in terms of energy preservation in eliminating redundant transmissions and in terms of coverage of the sensed area. In this work, an advanced sensor placement algorithm which depends on achieving a total coverage with the minimum possible number of sensors is presented.

Kawanlinderjit kaur gagneja et.al.[4] proposed a Heterogeneous sensor networks; Voronoi; Tabu; Clustering; Security. Heterogeneous Sensor Networks are more powerful and efficient than homogeneous sensor networks. It has been shown that homogeneous sensor networks perform poorly because of routine limits and scalability. In our research, we consider to use heterogeneous topology to securely route data in a wireless sensor network. The given area of interest is initially partitioned into Voronoi clusters, where low-end nodes make clusters with high-end nodes. Each cluster has just one high-end node and a number of low-end nodes. An effort has been made to provide a complete solution to sensor networks, by first applying clustering, then securing the proposed routing algorithm.

Kendall E. Nygard et. al.[5] developed a Heterogeneous sensor networks; Routing; Security; Key management, Clustering; Clustering, routing and security are essential for better performance of any sensor network. Providing inbuilt security to routing algorithms in sensor networks is essential since some of these sensor networks have applications in hostile environments. Researchers work either on a routing algorithm or on security. However, the security should be embedded to the design of a routing scheme. In this paper, initially we divide the given area of interest into Voronoi clusters and then apply a new Tabu heuristic to form more stable clusters. When some event occurs, a new efficient key management technique is applied with improved tree routing algorithm for data routing in heterogeneous sensor networks. It presented a new approach for HSNs, namely TV-SITR.

Chuan-Kang Ting et.al.[6] proposed a tabu search; meta heuristic algorithm; wireless sensor network; lifetime ; set kcover problem. Lifetime is a key issue in wireless sensor network (WSN) applications. An effective way to extend WSN lifetime is to partition the set of sensors into several covers and activate them alternately. More covers then lead to longer lifetime. Finding the maximum number of covers under full coverage constraint has been formulated as an NP-complete problem - the SET KCOVER problem. This study proposes the tabu search with random walk to deal with this problem. Simulation results show that the proposed algorithm outperforms existing heuristic algorithms and genetic algorithm in number of covers obtained. 
Sim Zaid et.al.[7] developed a Localization, wireless sensor networks (WSN)s, artificial neural networks (ANN)s, radio propagation pattern (RPP), anisotropic signal attenuation, cost and power efficiencies, robustness. It proposed a novel range-free localization algorithm for wireless sensor networks (WSN)s that is robust against the anisotropic signal attenuation induced by fading, shadowing, and interference, etc., present in any wireless channel, and hereby develop a new distance estimation (DE) approach able to efficiently derive distances' estimates in closed form.

Gurbinder Singh Brar et.al[8] developed a Wireless Sensor Networks; DSR; PEGASIS; PDORP; OD-PRRP; LEACH; Optimization; Hybridization Computation Model Energy consumption is one of the constraints in Wireless Sensor Networks (WSNs). The routing protocols are the hot areas to address quality-of-service (QoS) related issues viz. Energy consumption, network lifetime, network scalability and packet overhead. The key issue in WSN is that these networks suffer from the packet overhead, which is the root cause of more energy consumption and degrade the QoS in sensor networks which has used cache and directional transmission concept of both proactive and reactive routing protocols.

Abdelmorhit El Rhazi et.al [9] developed a Wireless sensor network, energy map, data collect, clustering methods, tabu search. Abstract-The main challenge in wireless sensor network deployment pertains to optimizing energy consumption when collecting data from sensor nodes. This paper proposes a new centralized clustering method for a data collection mechanism in wireless sensor networks, which is based on network energy maps and Quality-of-Service (QoS) requirements. The clustering problem is modeled as a hypergraph partitioning and its resolution is based on a tabu search heuristic.

S.Kazem Shekofteh et.al.[10] developed by a wireless sensor network, localization, tabu search, simulated annealing Sensor localization is a fundamental and crucial issue for wireless sensor networks operation and management. Accurate self-localization capability is highly desirable in wireless sensor network. A fundamental problem in distance based sensor network localization is whether a given sensor network is uniquely localizable or not. Flip ambiguity is a main problem that can make the sensor network not uniquely localized we have proposed and analysed a new algorithm comprised of two phases for the localization of WSN using a short-term memory TS method and simulated annealing.

\section{OPERATION BASED ROUTING PROTOCOLS}

\section{Negotiation Based Routing}

These protocols use high-level data descriptors called metadata in order to eliminate redundant data transmission through negotiations. The necessary decisions are based on available resources and local interactions. Sensor Protocols for Information via Negotiation (SPIN) is one of well-known Negotiation based routing protocol for WSN. The SPIN protocols are designed to disseminate the data of one sensor to all other sensors assuming these sensors are potential base-stations. Hence, the main idea of negotiation based routing in WSN is to suppress duplicate information and prevent redundant data from being sent to the next sensor or the base-station by conducting a series of negotiation messages before the real data transmission begins [32].

\section{Multipath Based Routing}

These protocols offer fault tolerance by having at least one alternate path (from source to sink) and thus, increasing energy consumption and traffic generation. These paths are kept alive by sending periodic messages. The path is switched whenever a better path is discovered. The primary path will be used until its energy is below the energy of the backup path. By means of this approach, the nodes in the primary path will not deplete their energy resources through continual use of the same route, thus achieving longer lifetime. A disadvantage for applications that require mobility on the nodes, is that the protocol is oriented to solve routing problem in static wireless networks. Hierarchical Power-aware Routing in Sensor Networks protocol enhances the reliability of WSN by using multipath routing. It is useful for delivering data in unreliable environments. The idea is to define many paths from source to sink and send through them the same sub-packets.

\section{Query Based Routing}

In these protocols, the destination nodes propagate a query for data (sensing task or interest) from the node through the network. The nodes containing this data send it back to the node that has initiated the query. Rumor routing protocol is one of the routing protocol used in the context of event notification. The approach does not flood the network with information about an event occurrence but only installs few paths in the network by sending out one or several agents.

\section{Location Based Routing}

In the protocols, the nodes are addressed by their location. Distances to next neighboring nodes can be estimated by signal strengths or by GPS receivers. Minimum Energy Communication Network protocol sets up and maintains a minimum energy network for wireless networks by utilizing low power GPS. Although, the protocol 
assumes a mobile network, it is best applicable to sensor networks, which are not mobile. Geographic Adaptive Fidelity (GAF) protocol is energy-aware location-based routing designed primarily for mobile ad hoc networks and can be applicable to sensor networks as well. GAF keeps energy by turning off unnecessary nodes in the network without affecting the level of routing fidelity. It forms a virtual grid for the covered area. Each node uses its GPS-indicated location to associate itself with a point in the virtual grid.

\section{Energy-Aware WSN Routing Protocol}

Energy Aware Routing is a reactive protocol to increase the lifetime of the network. This protocol maintains a set of paths instead of maintaining or reinforcing one optimal path. The maintenance and selection depends on a certain probability, which relays on how low the energy consumption of each path can be achieved. The protocol creates routing tables about the paths according to the costs. Localized flooding is performed by the destination node to maintain the paths alive.

\section{TABU SEARCH}

Tabu search is an adaptive search technique, using the best improvement local search as the basic ingredient. By allowing temporary solution degradation, tabu search avoids the search process being trapped into the local optimum. Two mechanisms, the short term memory and long term memory, can be applied to keep track of attributes of previously visited solutions and guide the tabu search process.

Tabu Search is a meta-heuristic that guides a local heuristic search procedure to explore the solution space beyond local optimality. One of the main components of Tabu Search is its use of adaptive memory, which creates a more flexible search behavior. Memory-based strategies are therefore the hallmark of tabu search approaches, founded on a quest for "integrating principles," by which alternative forms of memory are appropriately combined with effective strategies for exploiting them. A novel finding is that such principles are sometimes sufficiently potent to yield effective problem solving behavior in their own right, with negligible reliance on memory. Over a wide range of problem settings, however, strategic use of memory can make dramatic differences in the ability to solve problems. Pure and hybrid Tabu Search approaches have set new records in finding better solutions to problems in production planning and scheduling, resource allocation, network design, routing, financial analysis, telecommunications, portfolio planning, supply chain management, agent-based modelling, business process design, forecasting, machine learning, data mining, bio-computation, molecular design, forest management and resource planning, among many other areas.

The TS technique is rapidly becoming the method of choice for designing solution procedures for hard combinatorial optimization problems. A comprehensive examination of this methodology can be found in the book by Glover and Laguna (1997). Widespread successes in practical applications of optimization have spurred a rapid growth of the method as a means of identifying extremely high quality solutions efficiently. TS methods have also been used to create hybrid procedures with other heuristic and algorithmic methods, to provide improved solutions to problems.

The localization steps followed by using Tabu Search Algorithm are that it takes the results of Mobile Anchor Positioning as its input. The results of MAP, giving the approximate solution of the location of each sensor at each specified time instance is given as the input to the post optimization method. At any iteration it has to find a new solution by making local movements over the current solution. The possible solution of a node which was predicted by MAP algorithm is maintained in a tabu list. The average distance of neighbour nodes of the corresponding nodes are calculated. The difference between the location and the average distance of the node are calculated. If the solution is less than the average value then that value is considered as a best solution. The "next solution" is the best among all (or a subset of) possible solutions in the neighborhood in order to carry out the exploration process, the recently visited solutions are avoided. Tabu list is maintained. Therefore once a solution is visited, the movement from which it was obtained is considered as tabu. $\mathrm{N}(\Omega)$ will be changing along the exploration, so in a certain sense dynamic neighbourhood is compared to the previous local search algorithms where remains static. Typically there are two kinds of tabu lists, a long term memory and short term memory. Long term memory maintains the history through all the exploration process as a whole and a short term memory is to keep the most recently visited tabu movements. A movement with a tabu status (tabu movement) is avoided to be applied, unless it satisfies certain aspiration criteria. This aims to avoid falling into local optima. Tabu list size is fixed before the hand each element of the list belongs to it for a number of iterations bounded by given maximum and minimum values. Repeat the iterations until the stopping criteria are met.

\section{PROPOSED SYSTEM}

In this paper, the main goal is to construct a fast tabu search algorithm for computing solutions of good quality for large instances of the minmax problem in WSN. The maximum flow problem is intimately related to the minimum 
cut problem. A cut is ant set of directed arcs containing at least one arc in every path from the origin node to the destination node. In other words, if the arcs in the cut are removed, then flow from the origin to the destination is completely cut off. The cut value is the sum of the flow capacities in the origin-to-destination direction over all of the arcs in the cut. The minimum cut problem is to find the cut that has the minimum cut value over all possible cuts in the network. In order to find the minimum cut we will make use of the max-flow / min-cut theorem: for any network having a single origin node and a single destination node, the maximum possible flow from origin to destination equals cut value for all cuts in the network.

\section{CONCLUSION}

In this paper, it reviews on a WSN-TS algorithm to solve channel assignment problems in WSNs based on graph theory. It models the conflict graph for the feature of interferences from Inter-network and Intra-network to the connection links. It will investigate the problem of upgrading sensor nodes to maximize the flow rate. It will use the concept of path and Tabu to analyse the performance of system.

\section{REFFERENCES}

[1] Jaspreet kaur,'Tabu Search, Energy Efficiency, Clustering, Data Aggregation, ERA, WSNs.”,IEEE JOURNAL SENSOR,2015.

[2] Meng husi J.,"Wormhole attacks, Wireless Sensor Network, Quantum-inspired Tabu Search Algorithm”, IEEE JOURNAL SENSOR,2014.

[3] Amjad Osmani, "Design and Evaluation of New Intelligent Sensor Placement Algorithm to Improve Coverage Problem in Wireless Sensor Networks," Islamic

[4] Azad University, Saghez, Heterogeneous sensor networks; Voronoi; Tabu; Clustering", IEEE JOURNAL SENSOR, 2014.

[5] Kendall E. Nygard,'Heterogeneous sensor networks; Routing; Security; Key management, Clustering”, IEEE JOURNAL SENSOR,2013

[6] Chuan-Kang Ting," tabu search; metaheuristic algorithm; wirelesssensor network; lifetime ; set k-cover problem.", IEEE JOURNAL SENSOR,2013.

[7] Sim Zaid," Localization, wireless sensor networks (WSN)s, artificial neural networks (ANN)s, radio propagation pattern (RPP), anisotropic signal attenuation, cost and power efficiencies, robustness", IEEE JOURNAL SENSOR,2012.

[8] Gurbinder Singh Brar,"Wireless Sensor Networks; DSR; PEGASIS; PDORP; OD- PRRP; LEACH; Optimization", IEEE JOURNAL SENSOR,2012.

[9] Abdelmorhit El Rhazi and Samuel Pierre, "Wireless sensor network, energy map, data collect, clustering methods, tabu search." IEEE JOURNAL SENSOR, 2010.

[10] S.Kazem Shekofteh,"Wireless sensor network, localization, tabu search, simulated annealing" IEEE JOURNAL SENSOR, 2008

[11] MingJian Tang,"Wireless Sensor Networks, Multi-query Optimization," IEEE JOURNAL SENSOR, 2007.

[12] William Stallings, Wireless Communications and Networks, $2^{\text {nd }}$ ed., Pearson Education,Inc., 2004.

[13] T.S. Rappaport, Wireless Communications: Principles and Practice, Singapore: Pearson Education, Inc., 2002. 